\title{
Dephasing time in graphene due to interaction with flexural phonons
}

\author{
Konstantin S. Tikhonov *, $, 2,3,4, *$ Wei L.Z. Zhao ${ }^{*},{ }^{1,2, \dagger}$ and Alexander M. Finkel'stein ${ }^{1,2,5}$ \\ ${ }^{1}$ Department of Physics $\&$ Astronomy, Texas A $6 M$ University, College Station, TX 77843-4242, USA \\ ${ }^{2}$ Department of Condensed Matter Physics, The Weizmann Institute of Science, 76100 Rehovot, Israel \\ ${ }^{3}$ L. D. Landau Institute for Theoretical Physics, 117940 Moscow, Russia \\ ${ }^{4}$ Moscow Institute of Physics and Technology, 141700 Moscow, Russia \\ ${ }^{5}$ Institut für Nanotechnolsogie, Karlsruhe Institute of Technology, 76021 Karlsruhe, Germany
}

(Dated: June 17, 2021)

\begin{abstract}
We investigate decoherence of an electron in graphene caused by electron-flexural phonon interaction. We find out that flexural phonons can produce dephasing rate comparable to the electronelectron one. The problem appears to be quite special because there is a large interval of temperature where the dephasing induced by phonons can not be obtain using the golden rule. We evaluate this rate for a wide range of density $(n)$ and temperature $(T)$ and determine several asymptotic regions with temperature dependence crossing over from $\tau_{\phi}^{-1} \sim T^{2}$ to $\tau_{\phi}^{-1} \sim T$ when temperature increases. We also find $\tau_{\phi}^{-1}$ to be a non-monotonous function of $n$. These distinctive features of the new contribution can provide an effective way to identify flexural phonons in graphene through the electronic transport by measuring the weak localization corrections in magnetoresistance.
\end{abstract}

PACS numbers: 72.10.-d, 72.10.Di, 72.80.Vp

Introduction. The transport properties of graphene have attracted much attention [1] since the first discovery of this fascinating material [2]. It is promising for various applications due to its high charge mobility and unique heat conductivity. Theoretically, it was realized long ago [3-5] that these transport properties of freestanding (suspended) graphene are strongly influenced by flexural (out-of-plane) vibrational modes that deform the graphene sheet. From the experimental point of view, the effect of flexural phonons (FPs) was clearly observed in heat transport $[6,7]$. However, it is a more challenging task to identify the effect of flexural phonons in electronic transport $[8,9]$. This is because the contribution of electron-phonon interactions to momentum relaxation remains small even at high temperatures, with the main source of the relaxation being elastic impurities [10].

The dephasing rate $\tau_{\phi}^{-1}$, on the other hand, is a more suitable quantity for studying FPs, since static impurities do not cause dephasing. Usually, electron-electron interactions, [11-15] are considered the primary mechanism for dephasing. In this letter we discuss dephasing caused by the electron-flexural phonon (el-FP) interaction in graphene. It is the softness of the flexural mode and the coupling of an electron to two FPs simultaneously (see Fig. 1 for illustration) that make the contribution of FPs to $\tau_{\phi}^{-1}$ significant in a suspended sample, and at large enough densities comparable with the one caused by the electron-electron interaction. Because of the quadratic spectrum of FPs, $\omega_{k}=\alpha k^{2}$, they are much more populated as compared with in-plane phonons. In addition, the coupling to two FPs considerably increases the phase space available for inelastic processes as compared to the interaction with a single phonon. The point is that in graphene the Fermi momentum, $k_{F}$, is relatively small. As a result, the interaction of a single phonon with
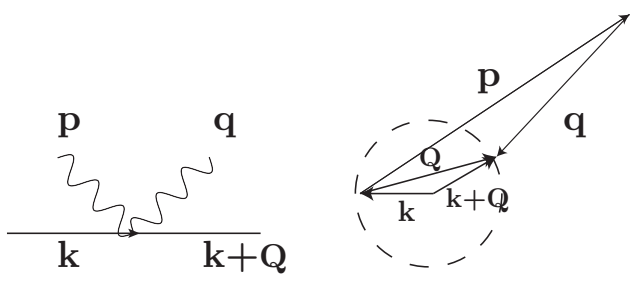

Figure 1. On the left: scheme of the el-FP interaction process, where the solid line represents an electron, and the wavy lines represent FPs. On the right: FPs can have momenta $\mathbf{p}, \mathbf{q}$ much larger than the transferred momentum Q. Under the conditions discussed in the paper, the scattering process is considered as semi-elastic.

electrons is determined by the Bloch-Grüneisen temperature, $T_{B G} \sim \omega_{2 k_{F}}$, rather than the temperature, when $T \gg T_{B G}[16]$. In such a case, one needs to exploit other scattering mechanisms to overcome the limitations induced by the smallness of $k_{F}$ [17]. In the case of el-FP interaction, coupling to two phonons radically changes the situation. Now only the transferred momentum should be small, while individually a FP may have a momentum much larger than $k_{F}$, up to the thermal momentum $q_{T}$.

Still, as we shall demonstrate, the problem of dephasing due to the el-FP interaction appears to be quite special, because the softness of FPs, i.e. unique smallness of $T_{B G}$, leads to the existence of a temperature range where dephasing rate cannot be obtained using the golden rule (GR). Rather, both the self-energy and the vertex processes [18] should be treated simultaneously. This results in a transition from $\tau_{\phi}^{-1} \sim T^{2}$ to $T$ with increasing temperature for the dephasing rate induced by FPs.

The electron-flexural phonon interaction. Lattice dynamics of the single-layer graphene can be described in terms of the displacement vector $\mathbf{u}=\left(u_{x}, u_{y}, h\right)$ [19]. 
Here $u_{x, y}$ describe the in-plane modes, while the out-ofplane displacement $h$ describes the flexural mode. The displacement vector leads to a non-linear strain tensor $u_{i j}=\frac{1}{2}\left(\partial_{i} u_{j}+\partial_{j} u_{i}+\partial_{i} h \partial_{j} h\right)$, where $(i, j)=(x, y)$ are spatial indices. The lattice modes interact with electrons through emergent scalar and vector potential fields $[20,21]$ :

$$
\begin{aligned}
\varphi & =g_{1}\left(u_{x x}+u_{y y}\right), \\
\mathbf{A}^{\alpha} & =s^{\alpha} g_{2} / v_{F}\left(u_{x x}-u_{y y},-2 u_{x y}\right),
\end{aligned}
$$

where $g_{1}=30 \mathrm{eV}, g_{2}=7.5 \mathrm{eV}[9]$ and $v_{F}$ is the Fermi velocity. Index $\alpha=K, K^{\prime}$ describes two valleys of the conducting electron band, and factor $s^{K / K^{\prime}}= \pm 1$ reflects the fact that the emergent vector potential $\mathbf{A}^{\alpha}$ respects the time reversal symmetry.

Thermal fluctuations of the lattice produce variations in the potentials. Averaging over lattice vibrations one finds the correlation functions of the potentials as

$$
\begin{aligned}
\langle\varphi(\mathbf{Q}, \Omega) \varphi(-\mathbf{Q},-\Omega)\rangle & =\phi(\mathbf{Q}, \Omega) \\
\left\langle A_{i}^{\alpha}(\mathbf{Q}, \Omega) A_{j}^{\beta}(-\mathbf{Q},-\Omega)\right\rangle & =s^{\alpha} s^{\beta} \mathcal{A}_{i j}(\mathbf{Q}, \Omega) .
\end{aligned}
$$

To proceed, we introduce the correlation function for FP

$$
\langle h(\mathbf{k}, \omega) h(-\mathbf{k},-\omega)\rangle \equiv H(k) 2 \pi \delta\left(\omega-\omega_{k}\right),
$$

where $H(k)=\frac{n\left(\omega_{k}\right)}{\rho \omega_{k}}$. In this equation, $n(\omega)$ is the Planck distribution function and $\rho$ is the mass density of the graphene sheet. One can propose the following form of the spectrum of the flexural phonon:

$$
\omega_{k}=\alpha k^{2} \Theta(k), \Theta(k)=\sqrt{1+Z^{-1}\left(q_{c} / k\right)^{\eta}},
$$

where $\Theta(k)$ describes a transition from the bare spectrum at high momentum to the renormalized spectrum $\sim k^{2-\eta / 2}$ in the low momentum limit. At $k<q_{c}(T)=$ $\frac{\sqrt{T \Delta_{c}}}{v_{F}}$ the quadratic spectrum for the flexural mode ceases to work due to anharmonicity. Here $\Delta_{c} \approx 18.7 \mathrm{eV}$ [5] reflects the energy scale of anharmonicity. The anharmonicity is related to the $h^{4}$-vertex, arising as a result of integrating out fast $u$-modes, which are coupled to $h$-mode [22]. Below we will exploit the value $Z \sim 2$, and take $\eta \approx 0.8$ from the numerical solution of the selfconsistent screening approximation theory [23, 24].

We consider graphene away from the Dirac point at chemical potential $\mu \gg T$. Besides $k_{F}$, the relevant momentum scales in the problem are: thermal momentum $q_{T}=\sqrt{T / \alpha} \approx 0.05 \sqrt{T[\mathrm{~K}]} / \AA$, and $q_{c}(T) \approx$ $0.01 \sqrt{T[\mathrm{~K}]} / \AA$ which signals the transition to the renormalized FP spectrum. From now on, we will concentrate on the realistic situation from the experimental viewpoint: $k_{F} \ll q_{T}$, i.e., $T \gg T_{B G}$. The Bloch-Grüneisen temperature $T_{B G}=\omega_{2 k_{F}} \approx 0.4 \Theta\left(2 k_{F}\right) n \mathrm{~K}$, where $n$ is the electronic density measured in units of $10^{12} \mathrm{~cm}^{-2}$.
Note $T_{B G}$ is extraordinarily small for all relevant densities. As we have already emphasized, see Fig. 1, the momentum transfer in the el-FP interaction is limited by $2 k_{F}$. Nevertheless, the extended structure of the correlation functions $\phi(\mathbf{Q}, \Omega)$ and $\mathcal{A}_{i j}(\mathbf{Q}, \Omega)$ enables electrons to have energy transfer exceeding the phonon en$\operatorname{ergy} \omega_{2 k_{F}}$.

Main tool to probe electronic coherence is magnetoresistance [25], which gives a direct access to the weak localization corrections to conductivity, controlled by the dephasing rate $\tau_{\phi}^{-1}$. The weak localization correction to conductivity in graphene can be written as $[26,27]$

$$
\Delta \sigma=-\frac{2 e^{2} D}{\pi} \sum_{l} \int d t C^{l}(-t / 2, t / 2),
$$

where $l$ sums over four Cooperon channels relevant for the magnetoresistance. Physically, $C^{l}(-t / 2, t / 2)$ represents the interference of a pair of time reversed trajectories in the channel $l$ that start at $-t / 2$ and return to the initial point at $t / 2$. More generally, the Cooperon matrix $C_{s_{1} s_{2}}^{l_{1} l_{2}}$ is labelled by two isospin numbers $s_{1,2}$ and two pseudospin numbers $l_{1,2}$. This matrix is diagonal in the pseudospin space even in the presence of interactions that preserve sublattice and valley indices. The Cooperon channels relevant for magnetoresistance are the isospin singlets, $C^{l} \equiv C_{00}^{l l}(l=0, x, y, z)$, that do not have gaps comparable with $\tau^{-1}$, the elastic scattering rate due to impurities. Therefore, we restrict ourselves to this subspace.

(a)

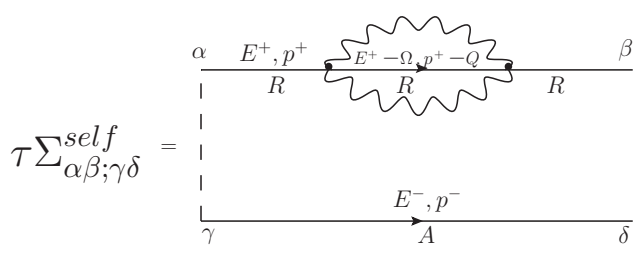

(b)

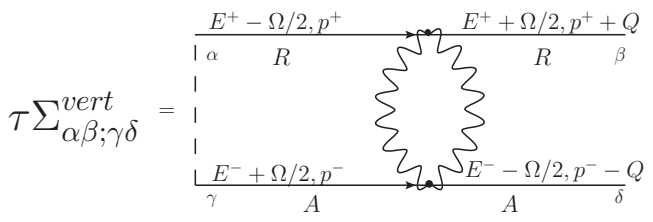

Figure 2. Diagrammatic representation of (a) the self-energy and (b) the vertex FP-contribution to the Cooperon; see also Fig. 2c in [28].

To include el-FP interaction into the Cooperon, one can write down a Bethe-Salpeter equation for a particular Cooperon channel $C^{l}$, see Fig. 2. In the following we will not solve the equation exactly, but instead, we will estimate the upper bound of the Cooperon decay rate $[29,30]$. We start by writing down an ansatz that reads as $[18]$

$$
C^{l}\left(t_{1}, t_{2}\right)=C_{0}^{l}\left(t_{1}-t_{2}\right) e^{-F^{l}\left(t_{1}, t_{2}\right)}
$$


Here $C_{0}^{l}(t)$ is the diffusion propagator describing the bare Cooperon, and $F^{l}\left(t_{1}, t_{2}\right)$ is a decay function characterizing the effect of the el-FP interaction [28].

Dephasing due to scalar potential fluctuations. For the scalar potential correlation function one obtains:

$$
\begin{aligned}
\phi(\mathbf{Q}, \Omega) & =\frac{1}{8} g_{1}^{2}(Q) \int\left(d^{2} \mathbf{p}\right)\left(d^{2} \mathbf{q}\right)[\mathbf{p} \cdot \mathbf{q}]^{2} \\
& \times H(p) H(q) \delta_{\mathbf{p}, \mathbf{q}}(\Omega, \mathbf{Q}),
\end{aligned}
$$

where $\delta_{\mathbf{p}, \mathbf{q}}(\Omega, \mathbf{Q}) \equiv \sum_{ \pm}(2 \pi)^{3} \delta\left(\Omega \pm \omega_{\mathbf{p}} \pm \omega_{\mathbf{q}}\right) \times$ $\delta(\mathbf{Q}-\mathbf{p}-\mathbf{q})$, and $\omega_{\mathbf{p}, \mathbf{q}}$ are given by Eq. (4). Here summation includes four different processes of emission/absorption of two FPs by an electron. The screened coupling constant $g_{1}(Q)=g_{1} \frac{Q}{Q+\varkappa}$, where $\varkappa=g_{e} N k_{F}$, $N=4$ is the spin-valley degeneracy in graphene, and $g_{e} \sim 1$ describes the renormalized Coulomb interaction [31]. Since each time an electron is coupled to two flexural phonons, $\phi$ describes a phonon loop and, therefore, in the momentum-frequency domain $\phi(\mathbf{Q}, \Omega)$ has a extended support rather than a $\delta$-function peak. As a result, the decay function for the scalar potential $F_{\phi}(t)$ (which is the same for all channels) can be expressed as a convolution of the three factors [28]: i) the correlation function $\phi(\mathbf{Q}, \Omega)$, ii) function $\mathcal{B}_{\phi}(\mathbf{Q})$, describing the ballistic electron's motion, and iii) factor $\mathcal{C}^{\phi}(\Omega, t)$, reflecting the relation between the self-energy and vertex diagrams:

$$
F_{\phi}(t)=t \int(d \mathbf{Q})(d \Omega) \phi(\mathbf{Q}, \Omega) \mathcal{B}_{\phi}(\mathbf{Q}) \mathcal{C}^{\phi}(\Omega, t) .
$$

Here,

$$
\mathcal{B}_{\phi}(\mathbf{Q})=\frac{2}{v_{F} Q}\left(1-\left(Q / 2 k_{F}\right)^{2}\right)^{1 / 2} \theta\left(2 k_{F}-Q\right),
$$

where the Heaviside theta function $\theta\left(2 k_{F}-Q\right)$ restricts momentum that can be exchanged between FPs and electrons. The factor $\mathcal{C}^{\phi}(\Omega, t)$ is equal to

$$
\mathcal{C}^{\phi}(\Omega, t)=1-\frac{\sin \Omega t}{\Omega t},
$$

and it describes the balance between the self-energy and vertex diagrams on Fig. 2. $\mathcal{C}^{\phi}$ is sensitive to dynamic aspect of the scattering event and, because of this, alters temperature dependence of $\tau_{\phi}^{-1}$.

The dephasing rate $\tau_{\phi}^{-1}$ is defined according to $F_{\phi}\left(\tau_{\phi}\right)=1$. The decay function can be most conveniently expressed as

$$
F_{\phi}(t)=c_{\phi}^{2} t T f(\mathcal{T}, \xi) \frac{T}{\mu},
$$

where $c_{\phi}=\frac{g_{1} / \rho \alpha^{2}}{2 \pi g_{e} N} \sim 1.2$ is dimensionless coupling constant and $f$ is a dimensionless function of two parameters: $\mathcal{T}=\alpha k_{F}^{2} t$ and $\xi=Z^{-1 / \eta} q_{c} / k_{F}$ [28]. Parameter $\xi$

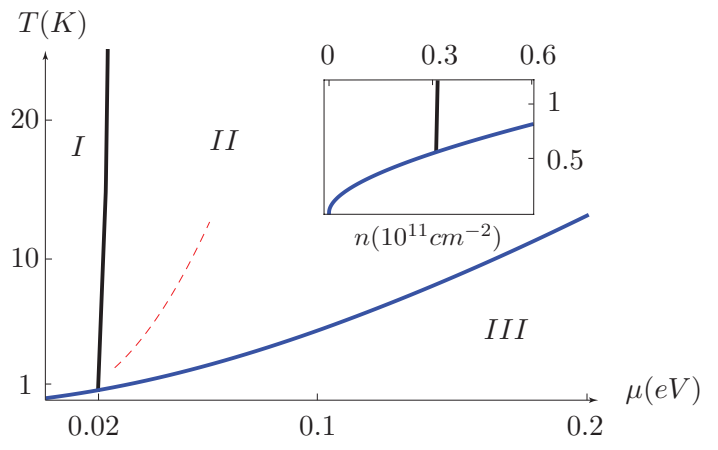

Figure 3. Phase diagram of the dephasing rate due to FPs with scalar coupling. The blue and black lines divide the whole $(T-\mu)$ plane into three regions, see the text for explanations. The blue line coincides with the maximum of the dephasing rate as a function of chemical potential at a fixed temperature, see Fig. (4). The red dashed line representing a fragment of $\xi=1$ is shown here for orientation. The inset is a zoom in of the intersection area of the blue and black lines plotted as a function of the electronic density.

originates from the renormalization of the $\mathrm{FP}$ spectrum described by $\Theta$ in Eq. (4); $\Theta\left(k_{F}\right)=\sqrt{1+\xi^{\eta}}$. At small $\mathcal{T}$ the function $f$ is linear in $\mathcal{T}$, and it saturates at $\mathcal{T} \gg 1$.

The results are illustrated with the help of Fig. 3, where regions I, II and III with a different dephasing rate behavior are indicated in the $(T-\mu)$ plane. The regions are divided in accord with the importance of the renormalized spectrum of the FP and the relative contributions of the self-energy and vertex diagrams. In region I, which is on the left of the black line (i.e., at small densities), the characteristic momenta of $p$ and $q$ in Eq. (7) do not exceed $q_{c}$. Therefore, the renormalization of the FP spectrum is important, and $\omega_{q} \sim q^{2-\eta / 2}$ should be used [32]. In region II, since the characteristic momenta of the FPs are larger than $q_{c}$, it suffices to use the quadratic spectrum for FPs. In region III, which is in the bottom part below the blue line, the dephasing time is long and only the self-energy diagram is important. Hence, the factor $\mathcal{C}^{\phi}$ reduces to 1 , and dephasing rate coincides with the out-scattering rate, $\tau_{\text {out }}^{-1}$, obtained from the golden rule [5]. (In this calculation, $q_{c}$ just provides an infrared cut-off.) Above the blue line, in regions I and II, both the self-energy and vertical diagrams are relevant, and the factor $\mathcal{C}^{\phi}(t)$ is important; see also [33]. Due to the two-phonon structure of the correlation function of the FP pairs participating in the inelastic process, the influence of this factor on the dephasing rate is rather non-trivial, so that one cannot expand $\mathcal{C}^{\phi}(t)$.

In Fig. 3, the blue and black lines have been found by matching the asymptotic behavior [28] of the dephasing rates deep in regions I, II and III. We introduce $\left(\mu_{0}, T_{0}\right)$, the values of the crossing point of the blue and black lines as characteristic scales: $\mu_{0} \sim \frac{\gamma}{c_{\phi}^{2}} \Delta_{c}$ and $T_{0} \sim \frac{\gamma}{c_{\phi}^{2}} \mu_{0}$. Here, we have introduced $\gamma=\frac{\alpha \Delta_{c}}{v_{F}^{2}} \sim 0.02$, which is a 
parameter describing the adiabaticity of the el-FP interaction. Under a given choice of parameters, it can be found numerically that $\mu_{0} \approx 0.02 \mathrm{eV}$ and $T_{0} \approx 0.6 \mathrm{~K}$. The dephasing rate in different regions can be expressed as

$$
\tau_{\phi}^{-1}(T)=\gamma T \times\left\{\begin{array}{cc}
0.48\left(\mu / \mu_{0}\right)^{\frac{4-\eta}{8-5 \eta}} & \text { I } \\
0.18 \sqrt{\mu / \mu_{0}} & \text { II } \\
0.24 \frac{T / T_{0}}{\mu / \mu_{0}} \log \xi^{-1} & \text { III } .
\end{array}\right.
$$

These expressions are obtained using asymptotic behavior of the function $f$ in Eq. (11) and, therefore, are only applicable far away from the borderlines. At low enough temperatures, $\mathcal{C}^{\phi}(t)=1$ and the function $f(\mathcal{T}, \xi)$ is independent of $\mathcal{T}$. Hence, $\tau_{\phi}^{-1} \sim T^{2}$ in region III, which is a GR result. At high temperatures the phonons contributing to the electronic dephasing become quasi-static and, consequently, the dephasing rate is smaller than the out-scattering rate $\tau_{\text {out }}^{-1}$. Unlike region III, in regions I and II the dephasing rate is determined by a non-GR expression, and is proportional to temperature, irrespective of $\eta$. The existence of the linear in $T$ regime is the main result of our paper.

By comparing the rates in regions II and III, one may conclude that there should be a maximum in the dephasing rate as a function of $\mu$. Indeed, as it is illustrated by Fig. 4 such a maximum exists. The line indicating the maximum essentially overlaps with the borderline between the regions I, II and the region III, which is illustrated by the blue line in Fig. 3 .

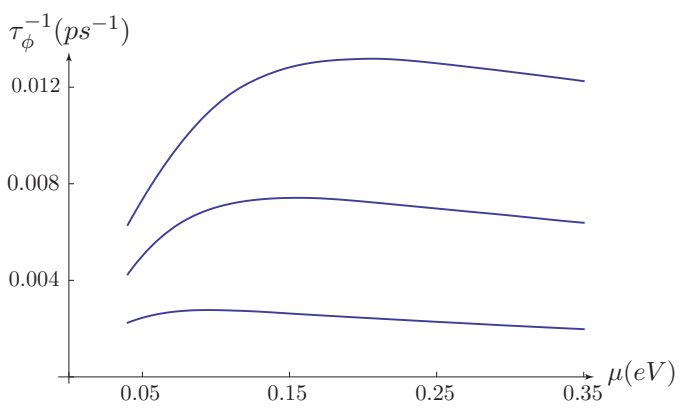

Figure 4. Dephasing rate as a function of the chemical potential at different temperatures. From top to buttom: T=15K, $10 \mathrm{~K}, 5 \mathrm{~K}$.

Dephasing due to vector potential fluctuations. Unlike the scalar potential, the dephasing rates induced by vector potential are different for different channels owing to the factor $\mathcal{C}_{l}^{A}(\Omega, t)=1+s_{l} \frac{\sin \omega t}{\omega t}$, where $s_{l}=-1$ for the intervalley Cooperons $(l=0, z)$ and $s_{l}=1$ for the intravalley Cooperons $(l=x, y)$. For the intervalley channels, the only relevant for the magnetoresistance at weak fields, the dephasing rate produced by the vector potential coupling is quite similar to its scalar counterpart, Eq. (12), with obvious modifications due to the change in the coupling constant and absence of screening for the vector potential [28].
Discussion. We have analysed the dephasing rate induced by FPs in graphene, and evaluated it for a wide range of $n$ and $T$ (see Fig. 3.) We determined several asymptotic regions with temperature dependence evolving from $\tau_{\phi}^{-1} \sim T^{2}$ to $\tau_{\phi}^{-1} \sim T$ when temperature increases. (See Fig. 4 in [28] for an illustration of the temperature dependence of the dephasing rate.) The transition to linear behavior in $T$ is related to the fact that at high temperatures phonons become slow on the timescale of $\tau_{\phi}$.

The measured dephasing rate in graphene is usually compared to the contribution induced by the electronelectron interaction, $\tau_{e e}^{-1}$, which is linear in $T$ for $T<$ $1 / \tau_{\text {tr }}$ [11]. However, the observed rate [12-14], when it is linear in $T$, always exceeds the theoretical estimation. In view of the linear dependence on $T$ of the FP's contribution to dephasing, it is reasonable to compare its value with $\tau_{e e}^{-1}$. In principle, it is a competition between two mechanisms, each determined by a small parameter: the adiabatic parameter $\gamma$ and sheet resistance $\rho_{\square}$ measured in units of the quantum resistance. We compare the dephasing rates at density $n=10^{12} \mathrm{~cm}^{-2}$ when the sheet resistance $\approx 0.5 k \Omega$. Under these conditions, both parameters $\gamma$ and $\rho_{\square}$ are of the same value. Combining the contributions arising from the scalar and vector potentials, we obtain $\tau_{F P}^{-1} / \tau_{e e}^{-1} \approx 0.2$.

The in-plane phonons generate a dephasing $\tau_{i n}^{-1}$ that at $T<T_{B G}^{i n}$ is negligible compared with $\tau_{F P}^{-1}$, while at $T>$ $T_{B G}^{i n}$ the rate $\tau_{i n}^{-1} \sim T$ is comparable with $\tau_{F P}^{-1}$. (Note that for in-plane phonons, a region of non-GR dephasing rate, analogous to region II, develops at temperatures $\gtrsim \mu$ that is too high to be relevant.) It is important that each of the three rates $\tau_{e e}^{-1}, \tau_{F P}^{-1}$, and $\tau_{i n}^{-1}$, has a distinct dependence on the chemical potential. While $\tau_{e e}^{-1}$ decreases with density, $\tau_{F P}^{-1} \propto \mu^{1 / 2}$ and $\tau_{i n}^{-1} \propto \mu$. This opens a way to identify each of these mechanisms by studying the magnetoresistance as a function of the chemical potential.

In our consideration, we had in mind suspended graphene. However, our result may also be relevant for supported samples so long as they are coupled to the substrate by weak Van der Waals forces [34]. One may expect that such a weak coupling does not provide an essential change in the phonon spectrum. Indeed, it is known that the phonon spectrum in graphene [35] and graphite [36] are practically identical for the corresponding branches. FPs in supported samples have been discussed recently in connection with the heat transport measurements in Refs. [6, 7]. Until now flexural phonons have been a delicate object to detect in electronic transport. We propose here to observe them through weaklocalization measurements.

Acknowledgements. The authors gratefully acknowledge A. Dmitriev, I. Gornyi, V. Kachorovskii, D. Khmelnitskii and A. Mirlin for the useful discussions and valuable criticism. The authors thank the members of the In- 
stitut für Theorie der Kondensierten Materie at KIT for their kind hospitality. A.F. is supported by the Alexander von Humboldt Foundation. The work is supported by the Paul and Tina Gardner fund for Weizmann-TAMU collaboration, and National Science Foundation grant NSF-DMR-100675.

* K.Tikhonov and W.Zhao contributed equally to this work.

* tikhonov@physics.tamu.edu

† wei.zhao@tamu.edu

[1] S. Das Sarma, S. Adam, E. H. Hwang, and E. Rossi, Reviews of Modern Physics 83, 407 (2011).

[2] A. H. Castro Neto, N. M. R. Peres, K. S. Novoselov, and A. K. Geim, Reviews of Modern Physics 81, 109 (2009).

[3] E. Mariani and F. von Oppen, Phys. Rev. Lett. 100, 076801 (2008).

[4] H. Ochoa, E. V. Castro, M. I. Katsnelson, and F. Guinea, Phys. Rev. B 83, 235416 (2011).

[5] I. V. Gornyi, V. Y. Kachorovskii, and A. D. Mirlin, Phys. Rev. B 86, 165413 (2012).

[6] A. A. Balandin, S. Ghosh, W. Bao, I. Calizo, D. Teweldebrhan, F. Miao, and C. N. Lau, Nano Letters 8, 902 (2008).

[7] J. H. Seol et al., Science 328, 213 (2010).

[8] K. I. Bolotin, K. J. Sikes, J. Hone, H. L. Stormer, and P. Kim, Phys. Rev. Lett. 101, 096802 (2008).

[9] E. V. Castro, H. Ochoa, M. I. Katsnelson, R. V. Gorbachev, D. C. Elias, K. S. Novoselov, A. K. Geim, and F. Guinea, Phys. Rev. Lett. 105, 266601 (2010).

[10] E. H. Hwang and S. Das Sarma, Phys. Rev. B 77, 115449 (2008), note that the phonon's contribution to resistivity is about $100 \mathrm{Ohm}$ on the background of few $\mathrm{kOhms}$.

[11] B. L. Altshuler, A. G. Aronov, and D. E. Khmelnitsky, Journal of Physics C-Solid State Physics 15, 7367 (1982).

[12] X. Wu, X. Li, Z. Song, C. Berger, and W. A. de Heer, Phys. Rev. Lett. 98, 136801 (2007).

[13] F. V. Tikhonenko, A. A. Kozikov, A. K. Savchenko, and R. V. Gorbachev, Phys. Rev. Lett. 103, 226801 (2009).

[14] M. B. Lundeberg and J. A. Folk, Phys. Rev. Lett. 105, 146804 (2010).

[15] J. Jobst, D. Waldmann, I. V. Gornyi, A. D. Mirlin, and H. B. Weber, Phys. Rev. Lett. 108, 106601 (2012).

[16] D. K. Efetov and P. Kim, Phys. Rev. Lett. 105, 256805
(2010).

[17] J. C. W. Song, M. Y. Reizer, and L. S. Levitov, Phys. Rev. Lett. 109, 106602 (2012).

[18] J. von Delft, F. Marquardt, R. A. Smith, and V. Ambegaokar, Phys. Rev. B 76, 195332 (2007).

[19] P. M. Chaikin and T. C. Lubensky, Principles of condensed matter physics, Vol. 1 (Cambridge Univ Press, 2000).

[20] H. Suzuura and T. Ando, Phys. Rev. B 65, 235412 (2002).

[21] J. L. Mañes, Phys. Rev. B 76, 045430 (2007).

[22] D. R. Nelson and L. Peliti, Journal De Physique 48, 1085 (1987).

[23] P. Le Doussal and L. Radzihovsky, Phys. Rev. Lett. 69, 1209 (1992).

[24] K. V. Zakharchenko, R. Roldan, A. Fasolino, and M. I. Katsnelson, Phys. Rev. B 82, 125435 (2010).

[25] B. L. Altshuler, D. Khmelnitzkii, A. I. Larkin, and P. A. Lee, Phys. Rev. B 22, 5142 (1980).

[26] B. L. Altshuler, A. G. Aronov, and D. E. Khmelnitsky, Solid State Communications 39, 619 (1981).

[27] E. McCann, K. Kechedzhi, V. I. Fal'ko, H. Suzuura, T. Ando, and B. L. Altshuler, Phys. Rev. Lett. 97, 146805 (2006).

[28] See Supplemental Material for the diagrammatic calculation, evaluation and aymptotic properties of the decay function, which includes Refs.[37, 38].

[29] W. Eiler, Journal of Low Temperature Physics 56, 481 (1984).

[30] F. Marquardt, J. von Delft, R. A. Smith, and V. Ambegaokar, Phys. Rev. B 76, 195331 (2007).

[31] V. N. Kotov, B. Uchoa, V. M. Pereira, F. Guinea, and A. H. Castro Neto, Reviews of Modern Physics 84, 1067 (2012).

[32] We, however, neglect the $h^{4}$-vertex corrections.

[33] G. Montambaux and E. Akkermans, Phys. Rev. Lett. 95, 016403 (2005).

[34] A. K. Geim and I. V. Grigorieva, Nature 499, 419 (2013).

[35] D. L. Nika, E. P. Pokatilov, A. S. Askerov, and A. A. Balandin, Phys. Rev. B 79, 155413 (2009).

[36] R. Al-Jishi and G. Dresselhaus, Phys. Rev. B 26, 4514 (1982).

[37] A. G. Aronov, A. D. Mirlin, and P. Wölfle, Physical Review B 49, 16609 (1994).

[38] A. G. Aronov and P. Wölfle, Phys. Rev. B 50, 16574 (1994). 


\title{
Supplemental Material for "Dephasing time in graphene due to interaction with flexural phonons"
}

\author{
Wei L.Z. Zhao, ${ }^{1,2}$ Konstantin S. Tikhonov, ${ }^{1,2,3,4}$ and Alexander M. Finkel'stein ${ }^{1,2,5}$ \\ ${ }^{1}$ Department of Physics \& Astronomy, Texas AEMM University, College Station, TX 77843-4242, USA \\ ${ }^{2}$ Department of Condensed Matter Physics, The Weizmann Institute of Science, 76100 Rehovot, Israel \\ ${ }^{3}$ L. D. Landau Institute for Theoretical Physics, 117940 Moscow, Russia \\ ${ }^{4}$ Moscow Institute of Physics and Technology, 141700 Moscow, Russia \\ ${ }^{5}$ Institut für Nanotechnolsogie, Karlsruhe Institute of Technology, 76021 Karlsruhe, Germany
}

(Dated: June 17, 2021)

\section{DIAGRAMMATIC CALCULATION OF THE DECAY FUNCTION}

The decay function $F^{l}$ defined in Eq. (6m) ('m' refers to the main text) can be illustrated with the following diagram:

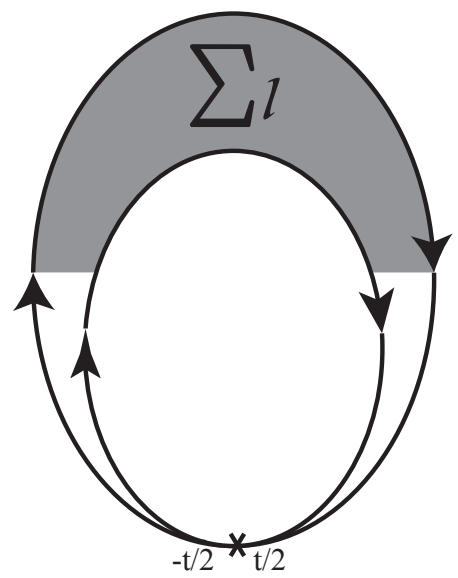

FIG. 1: Diagrammatic representation of the decay function $F(t)$. Here $\Sigma_{l}=\Sigma_{l}^{\text {self }}+\Sigma_{l}^{\text {vert }}$.

To the lowest order in the interaction propagators, the expression for $F^{l}$ can be obtained as [1]

$$
F^{l}\left(t_{1}, t_{2}\right) \simeq-\frac{C_{1}^{l}\left(t_{1}, t_{2}\right)}{C_{0}^{l}\left(t_{1}-t_{2}\right)},
$$

where $C_{1}^{l}$ is the first order correction to the Cooperon in terms of the interactions propagators. We perform the diagrammatic calculation with the use of the matrix Greens function $G_{\alpha \beta}^{R / A}(\epsilon, \mathbf{p})=\frac{1}{2} \frac{\delta_{\alpha \beta}+(\boldsymbol{\Sigma} \cdot \hat{\mathbf{p}})_{\alpha \beta}}{\epsilon \pm i /(2 \tau)-v_{F} p}$. Here we have introduced iso-pseudospin basis [2], spanned by two sets of mutually commuting matrices, isospin- $\Sigma$ and pseudospin$\Lambda:$

$$
\begin{aligned}
& \Sigma_{0}, \Sigma_{x}=\Pi_{z} \otimes \sigma_{x}, \Sigma_{y}=\Pi_{z} \otimes \sigma_{y}, \Sigma_{z}=\Pi_{0} \otimes \sigma_{z}, \\
& \Lambda_{0}, \Lambda_{x}=\Pi_{x} \otimes \sigma_{z}, \Lambda_{y}=\Pi_{y} \otimes \sigma_{z}, \Lambda_{z}=\Pi_{z} \otimes \sigma_{0},
\end{aligned}
$$

where $\Sigma_{0}, \Lambda_{0}$ are unit matrices and $\Pi_{x, y, z}$ and $\sigma_{x, y, z}$ are Pauli matrices acting on valley and sublattice spaces respectively. For each channel, the contribution of the el-FP interaction can be separated into the scalar and vector potential ones. Thus, one can write $F^{l}=F_{\phi}^{l}+F_{A}^{l}$. We concentrate first on dephasing caused by the scalar potential fluctuation $\phi(\mathbf{Q}, \Omega)$. The calculation for the vector potential contribution goes along the similar lines, and results are presented in the end of this Section. Due to the interaction propagators, one can write [1]

$$
\begin{aligned}
C_{1}^{l}\left(t_{1}, t_{2}\right)= & \int(d Q)(d \Omega)(d \tilde{q})(d \tilde{\omega}) e^{-i \tilde{\omega} t_{12}}\left[C_{0}^{l}(\tilde{q}, \tilde{\omega}) \Sigma_{Q, \tilde{q}}^{l, s e l f}(\tilde{\omega}, \Omega) C_{0}^{l}(\tilde{q}, \tilde{\omega})\right. \\
& \left.+e^{i \Omega \tau_{12}} C_{0}^{l}(\tilde{q}, \tilde{\omega}-\Omega) \Sigma_{Q, \tilde{q}}^{l, v e r t}(\tilde{\omega}, \Omega) C_{0}^{l}(\tilde{q}, \tilde{\omega}+\Omega)\right]
\end{aligned}
$$


where $t_{12}=t_{1}-t_{2}$ and $\tau_{12}=t_{1}+t_{2}$. Here, the effect of the interaction is separated into the self-energy and vertex (vertical) contributions, denoted as $\Sigma^{\text {self } / \text { vert }}$; see Fig. 2. The self-energy and vertex contribution of the channel $l$ are defined in the iso-pseudospin basis via $\Sigma^{l, \text { self } / \text { vert }} \equiv\left(\Sigma_{y} \Lambda_{y} \Lambda_{l}\right)_{\alpha \beta} \Sigma_{\alpha \beta ; \gamma \delta}^{\text {self } / \text { vert, }}\left(\Sigma_{y} \Lambda_{l} \Lambda_{y}\right)_{\delta \gamma}$ (summation for Greek letters is implied; indices $\phi(A)$ are omitted). Here, the self-energy contribution, see Fig. 2(a), can be written as

(a)

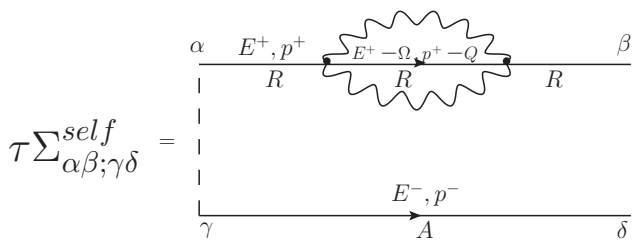

(b)

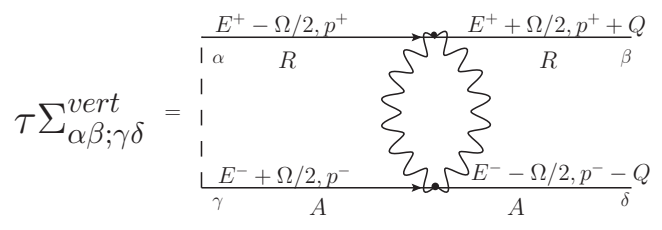

(c)

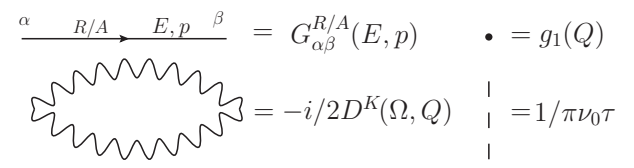

FIG. 2: Diagrammatic representation of (a) the self-energy contribution and (b) the vertex contribution. (c) Diagrammatic dictionary for the various objects involved: $E^{ \pm}=\mu \pm \tilde{\omega} / 2$ and $p^{ \pm}=k_{F} \pm \tilde{q} / 2 ; \nu_{0}$ is the density of states at Fermi energy, and $g_{1}(Q)$ is the coupling constant with screening included.

$$
\begin{aligned}
& \tau \Sigma_{\alpha \beta ; \gamma \delta,}^{s e l f, \phi}(\tilde{q}, Q, \tilde{\omega}, \Omega)=-\frac{g(Q)^{2}}{\pi \nu_{0} \tau} \int\left(d^{2} p\right)\left(-\frac{i}{2} D^{K}(Q, \Omega)\right) \times \\
& \left\{\left[G^{R}\left(p^{+}, E^{+}\right) G^{R}\left(p^{+}-Q, E^{+}-\Omega\right) G^{R}\left(p^{+}, E^{+}\right)\right]_{\alpha \beta} G^{A}\left(p^{-}, E^{-}\right)_{\gamma \delta}\right. \\
& \left.+G^{R}\left(p^{+}, E^{+}\right)_{\alpha \beta}\left[G^{A}\left(p^{-}, E^{-}\right) G^{A}\left(p^{-}-Q, E^{-}-\Omega\right) G^{A}\left(p^{-}, E^{-}\right)\right]_{\gamma \delta}\right\},
\end{aligned}
$$

where $E^{ \pm}=\mu \pm \tilde{\omega} / 2, p^{ \pm}=k_{F} \pm \tilde{q} / 2$. Note that arguments $\tilde{q}$ and $\tilde{\omega}$ are related to the propagation of the Cooperon, while $Q$ and $\Omega$ describe the interactions with FPs causing the dephasing. The vertex contribution, see Fig. 2(b), is

$$
\begin{aligned}
\tau \Sigma_{\alpha \beta ; \gamma \delta}^{v e r t, \phi}(\tilde{q}, Q, \tilde{\omega}, \Omega) & =-\frac{g(Q)^{2}}{\pi \nu_{0} \tau} \int\left(d^{2} p\right)\left(-\frac{i}{2} D^{K}(Q, \Omega)\right) \times \\
& {\left[G^{R}\left(p^{+}, \varepsilon^{+}-\Omega\right) G^{R}\left(p^{+}+Q, \varepsilon^{+}\right)\right]_{\alpha \beta}\left[G^{A}\left(p^{-}, \varepsilon^{-}+\Omega\right) G^{A}\left(p^{-}-Q, \varepsilon^{-}\right)\right]_{\gamma \delta} }
\end{aligned}
$$

where $\varepsilon^{ \pm}=E^{ \pm} \pm \Omega / 2$. Due to the softness of FPs, the integrals in Eq. (3) converge at small frequencies $\Omega \ll T$. Note that the assumption that $\Omega \ll T$ depends crucially on the fact that $\tau_{\phi}^{-1} \ll T$ which has been checked a posteriori. Therefore, when calculating the effect of interaction on the Cooperon propagators, one may take only the classical (Keldysh) component of the interaction, $D_{\phi}^{K}(\mathbf{Q}, \Omega)=-2 i \phi(\mathbf{Q}, \Omega)$, and send $n\left(\omega_{k}\right)$ in $H(k)$ to its classical limit, $\frac{T}{\omega_{k}}$. If the full quantum problem is considered, one needs to use $\frac{1}{\sinh \left(\omega_{k} / T\right)}$, in order to incorporate Pauli principle due the presence of other electrons [3]. This leads to the same result for $T \gg \omega_{k}$, but ensures that quantum fluctuations do not lead to dephasing at zero temperature.

In Eq. (3), the Cooperon variables $(\tilde{q}, \tilde{\omega})$ are small comparing to the electronic scales determining the el-FP interaction process, i.e., $\tilde{q} \ll k_{F}$ and $\tilde{\omega} \ll \mu$. Therefore, one can drop out $(\tilde{q}, \tilde{\omega})$ dependences from the interaction propagators $\Sigma$. Furthermore, as far as $T \gg T_{B G}$, the typical momentum transfer $Q \sim 2 k_{F} \gg 1 / v_{F} \tau$. Therefore, dephasing can also be calculated assuming that electron's motion during the interaction event is ballistic. After a simple calculation, one concludes for scalar potential

$$
\Sigma_{\phi}^{l, \text { self }}=-\Sigma_{\phi}^{l, v e r t}=\Sigma_{\phi}(Q, \Omega),
$$


where $\Sigma_{\phi}(Q, \Omega) \equiv \phi(\mathbf{Q}, \Omega) \mathcal{B}_{\phi}(Q)$. Here $\phi(\mathbf{Q}, \Omega)$ is defined in Eq. $(7 \mathrm{~m})$, and $\mathcal{B}_{\phi}(Q)$ is defined in Eq. (9m). Plugging these results back to Eq. (1) leads to Eq. (8m).

In the case of vector potential coupling, one calculates a diagram similar to that in Fig. 2, and gets

$$
\Sigma_{A}^{l, \text { self }}=s_{l} \Sigma_{A}^{l, v e r t}=\Sigma_{A}^{i j}(Q, \Omega) \delta_{T}^{i j}(Q),
$$

where $\delta_{T}^{i j}(Q)=\delta^{i j}-Q^{i} Q^{j} / Q^{2}$ and $\Sigma_{A}^{i j}(Q, \Omega)=v_{F}^{2} \mathcal{A}^{i j}(\mathbf{Q}, \Omega) \mathcal{B}_{A}(Q)$. Here, the vector potential correlation function

$$
\begin{aligned}
\mathcal{A}_{i j}(\mathbf{Q}, \Omega) & =\frac{\hat{\mathbf{n}}_{i} \hat{\mathbf{n}}_{j}}{8}\left(g_{2} / v_{F}\right)^{2} \int\left(d^{2} \mathbf{p}\right)\left(d^{2} \mathbf{q}\right) p^{2} q^{2} \\
& \times H(p) H(q) \delta_{\mathbf{p}, \mathbf{q}}(\Omega, \mathbf{Q}),
\end{aligned}
$$

where $\delta_{\mathbf{p}, \mathbf{q}}(\Omega, \mathbf{Q})=\sum_{ \pm}(2 \pi)^{3} \delta\left(\Omega \pm \omega_{\mathbf{p}} \pm \omega_{\mathbf{q}}\right) \delta(\mathbf{Q}-\mathbf{p}-\mathbf{q})$ and the summation includes four different processes of emission/absorption of two FPs by an electron. Also, the factor describing the ballistic motion of the electrons

$$
\mathcal{B}_{A}(Q)=\frac{2}{v_{F} Q}\left(1-\left(Q / 2 k_{F}\right)^{2}\right)^{-1 / 2} \theta\left(2 k_{F}-Q\right) .
$$

The corresponding decay function can be rendered as (compare with Eq. (8m))

$$
F_{A}^{l}(t)=t \int(d \mathbf{Q})(d \Omega) v_{F}^{2} \mathcal{A}^{i j}(\mathbf{Q}, \Omega) \delta_{T}^{i j}(Q) \mathcal{B}_{A}(Q) \mathcal{C}_{l}^{A}(\Omega, t)
$$

where

$$
\mathcal{C}_{l}^{A}(\Omega, t)=1+s_{l} \frac{\sin \Omega t}{\Omega t},
$$

The intervalley Cooperons are coupled to the vector potential field of the opposite signs. Thus, $s_{l}= \pm 1$ for intra/intervalley Cooperons. Further on, one can resolve the transverse delta function $\delta_{T}^{i j}$ and get

$$
F_{A}^{l}(t)=t \int \sin ^{3} \hat{Q}(d \mathbf{Q})(d \Omega) \mathcal{A}(\mathbf{Q}, \Omega) \mathcal{B}_{A}(Q) \mathcal{C}_{l}^{A}(\Omega, t),
$$

where

$$
\mathcal{A}(\mathbf{Q}, \Omega)=\frac{g_{2}^{2}}{8} \int\left(d^{2} \mathbf{p}\right)\left(d^{2} \mathbf{q}\right) p^{2} q^{2} H(\mathbf{p}) H(\mathbf{q}) \delta_{\mathbf{p}, \mathbf{q}}(\Omega, \mathbf{Q})
$$

\section{EVALUATION OF THE DECAY FUNCTION}

\section{Scalar potential coupling}

Let us now evaluate the integral in Eq. (8m) explicitly. It is convenient to use the time representation for the energy delta-function:

$$
\sum_{ \pm}(2 \pi) \delta\left(\Omega \pm \omega_{\mathbf{p}} \pm \omega_{\mathbf{q}}\right)=4 \int d \tau \cos \left(\omega_{p} \tau\right) \cos \left(\omega_{q} \tau\right) \exp [-i \Omega \tau]
$$

After this, one can integrate Eq. (8m) in frequency $\Omega$, using

$$
\int(d \Omega) e^{-i \Omega \tau}\left(1-\frac{\sin \Omega t}{\Omega t}\right)=\frac{1}{t} \Xi_{-}(\tau / t),
$$

where

$$
\Xi_{ \pm}(s) \equiv \delta(s) \pm \frac{1}{2} \theta(1-|s|) .
$$


The next step is to make the integral dimensionless by introducing $\tau=$ st and dimensionless $2 \mathrm{D}$ vectors $\mathbf{x}, \mathbf{y}, \mathbf{z}=\mathbf{p} / k_{F}, \mathbf{q} / k_{F}, \mathbf{Q} / k_{F}$. Using the expression for $H(q)$ in the classical limit, $H(q)=\frac{T}{\rho \omega_{q}^{2}}\left(\omega_{q}\right.$ is defined in Eq. $(4 \mathrm{~m}))$, we obtain the decay function as given in Eq. (11m)

$$
F_{\phi}(t)=c_{\phi}^{2} t T f_{\phi}\left(\alpha k_{F}^{2} t, Z^{-1 / \eta} q_{c} / k_{F}\right) \frac{T}{\mu} .
$$

Here,

$$
f_{\phi}(\mathcal{T}, \xi)=4 \pi^{2} \int(2 \pi)^{2}(d \mathbf{z})(d \mathbf{x})(d \mathbf{y}) \delta(\mathbf{z}-\mathbf{x}-\mathbf{y}) S(z) \frac{(\mathbf{x} \cdot \mathbf{y})^{2}}{x^{4} y^{4}} \int \Xi_{-}(s) d s \frac{\cos \left(\mathcal{T} s x^{2} \Theta_{\xi}(x)\right) \cos \left(\mathcal{T} s y^{2} \Theta_{\xi}(y)\right)}{\Theta_{\xi}^{2}(x) \Theta_{\xi}^{2}(y)}
$$

with $\mathcal{T}=\alpha k_{F}^{2} t, \xi=Z^{-1 / \eta} q_{c} / k_{F}, \Theta_{\xi}(x)=\sqrt{1+(x / \xi)^{-\eta}}$, and

$$
S_{\phi}(z)=\left(\frac{z}{1+z /\left(g_{e} N\right)}\right)^{2} \sqrt{1-(z / 2)^{2}} \theta(2-z),
$$

which is the product of the screening and chiral factors. Here $N=4$ is the spin-valley degeneracy in graphene, and $g_{e}$ describes the renormalized Coulomb interaction.

To proceed, it is convenient to make some transformations in Eq. (18). First, we integrate out $s$ exactly, using the relation

$$
\int \Xi_{ \pm}(s) d s \cos (s a) \cos (s b)=\Xi_{ \pm}(a, b)
$$

with

$$
\Xi_{ \pm}[a, b]=1 \pm \frac{a \sin a \cos b-b \sin b \cos a}{a^{2}-b^{2}}
$$

This gives

$$
f_{\phi}(\mathcal{T}, \xi)=(2 \pi)^{4} \int(d \mathbf{z})(d \mathbf{x})(d \mathbf{y}) \delta(\mathbf{z}-\mathbf{x}-\mathbf{y}) S_{\phi}(z) \frac{(\mathbf{x} \cdot \mathbf{y})^{2}}{x^{4} y^{4}} \frac{\Xi_{-}\left[\mathcal{T} \Theta_{\xi}(x) x^{2} s, \mathcal{T} \Theta_{\xi}(y) y^{2} s\right]}{\Theta_{\xi}^{2}(x) \Theta_{\xi}^{2}(y)}
$$

The final step is to resolve the delta-function in the above expression for $\mathbf{y}$ which yields

$$
y=\sqrt{z^{2}+x^{2}-2 z x \cos \psi}
$$

where $\psi$ is the angle between $\mathbf{z}$ and $\mathbf{x}$. As a result, one finally obtains

$$
f_{\phi}(\mathcal{T}, \xi)=\int_{0}^{2} d z S_{\phi}(z) \int_{0}^{\infty} d x \int_{0}^{2 \pi} \frac{d \psi}{2 \pi} \frac{(z \cos \psi-x)^{2}}{x y^{4}} \frac{\Xi_{-}\left[\mathcal{T} \Theta_{\xi}(x) x^{2}, \mathcal{T} \Theta_{\xi}(y) y^{2}\right]}{\Theta_{\xi}^{2}(x) \Theta_{\xi}^{2}(y)}
$$

\section{Vector potential coupling}

We define

$$
F_{A}^{l}(t) \equiv c_{A}^{2} t T f_{A}^{l}\left(\alpha k_{F}^{2} t, Z^{-1 / \eta} q_{c} / k_{F}\right) \frac{T}{\mu},
$$

where $c_{A}$ is the dimensionless el-FP coupling constant for the vector potential.

$$
\text { Intervalley channels }(l=0, z)
$$

Since $s_{l}=-1$, the decay function for the intervalley Cooperons is similar with that for the scalar potential. Without providing further details, we conclude that

$$
f_{A}^{l}(\mathcal{T}, \xi)=\int_{0}^{2} d z S_{A}(z) \int_{0}^{\infty} d x \int_{0}^{2 \pi} \frac{d \psi}{2 \pi} \frac{1}{x y^{2}} \frac{\Xi_{-}\left[\mathcal{T} \Theta_{\xi}(x) x^{2}, \mathcal{T} \Theta_{\xi}(y) y^{2}\right]}{\Theta_{\xi}^{2}(x) \Theta_{\xi}^{2}(y)}
$$


where $y$ has been defined by Eq. (23), and

$$
S_{A}(z)=\frac{1}{2}\left(1-(z / 2)^{2}\right)^{-1 / 2} \theta(2-z) .
$$

Note that $S_{A}(z)$ includes only the chiral factor since screening does not affect the vector potential coupling constant.

$$
\text { Intravalley channels }(l=x, y)
$$

For the intravalley channels, $s_{l}=1$. Correspondingly $\Xi_{-}$has to be changed to $\Xi_{+}$:

$$
f_{A}^{l}(\mathcal{T}, \xi)=\int_{0}^{2} d z S_{A}(z) \int_{0}^{\infty} d x \int_{0}^{2 \pi} \frac{d \psi}{2 \pi} \frac{1}{x y^{2}} \frac{\Xi_{+}\left[\mathcal{T} \Theta_{\xi}(x) x^{2}, \mathcal{T} \Theta_{\xi}(y) y^{2}\right]}{\Theta_{\xi}^{2}(x) \Theta_{\xi}^{2}(y)} .
$$

\section{ASYMPTOTIC PROPERTIES OF THE DECAY FUNCTION AND THE PHASE DIAGRAM}

The analytical expressions obtained in the previous sections allow us to determine $\tau_{\phi / A}$ at arbitrary temperature $T$ and chemical potential $\mu$. (In the end, the dephasing times $\tau_{\phi / A}$ are defined as solution of the equations $F_{\phi / A}\left(\tau_{\phi / A}\right)=1$.) We have developed numerical procedure, which exploits these equations to calculate corresponding $\tau_{\phi}(T, \mu)$ dependencies. Before presenting general results, let us concentrate on the properties of the functions $f_{\phi / A}(\mathcal{T}, \xi)$ in the analytically accessible regimes.

\section{Scalar potential coupling}

Large $\xi$

For $\xi \gg 1$, one may identify three asymptotic regions depending on $\mathcal{T}$. When $\mathcal{T} \ll \xi^{-2}$, the integral in $f_{\phi}(\mathcal{T}, \xi)$ is dominated by the quadratic spectrum where $\Theta_{\xi}(x)=1$ (refer to Eq. (24)), while at $\xi^{-2} \ll \mathcal{T} \ll \xi^{-\eta / 2}$ the integral is dominated by the part of spectrum where $\Theta_{\xi}(x)=(x / \xi)^{-\eta / 2}$. Thus, for not too large $\mathcal{T}\left(\mathcal{T} \ll \xi^{-\eta / 2}\right)$, one may assume that the spectrum is homogeneous, and put $\Theta_{\xi}(x)=(x / \xi)^{b}$. Then the function $f_{\phi}$ reads:

$$
f_{\phi}(\mathcal{T}, \xi)=\frac{\xi^{4 b}}{2 \pi} \int_{0}^{2} d z S_{\phi}(z) \int_{0}^{\infty} d x \int_{0}^{2 \pi} d \psi \frac{(z \cos \psi-x)^{2}}{x^{1+2 b} y^{4+2 b}} \Xi_{-}\left[\mathcal{T}(x / \xi)^{b} x^{2}, \mathcal{T}(y / \xi)^{b} y^{2}\right]
$$

We obtain,

$$
f_{\phi}(\mathcal{T}, \xi)=c(b) \xi^{\frac{6 b}{2+b}} \mathcal{T}^{\frac{2+4 b}{2+b}},
$$

with $c(b)=\frac{M_{b} N_{\phi}}{2+b}$, where $N_{\phi}=\int_{0}^{2} d z S_{\phi}(z)=0.89$ (assuming $g_{e} \approx 1$ ), and

$$
M_{b}=\int_{0}^{\infty} u^{-\frac{5 b+4}{b+2}} \Xi_{-}(u, u) d u=2^{\frac{3 b}{b+2}} \Gamma\left(-\frac{5 b+4}{b+2}\right) \sin \frac{(5 b+4) \pi}{(b+2) 2} .
$$

More specifically, for $b=-\eta / 2, c_{I}^{\eta} \equiv c(-\eta / 2)=0.03$, when $\eta=0.8$. For $b=0, c_{I I} \equiv c(0)=\frac{\pi}{8} N_{\phi}$. The scaling expression in Eq. (30) can be achieved due to the fact that one can neglect all the $z$ dependences except for $S_{\phi}(z)$ in Eq. (29). Then, $y \simeq x, \psi$ integral gives $2 \pi$, and the remaining integral over $x$ leads to Eq. (30). The obtained asymptotes will describe non-golden rule behavior for the decay function, in the region I $(b=-\eta / 2)$ and II $(b=0)$. Note that for $b=0, f_{\phi}=c_{I I} \mathcal{T}$.

Next, for $\mathcal{T} \gg \xi^{-\eta / 2}$, the golden rule regime holds and the vertex diagram is not important any more. In this case, one can put $\Xi_{-} \rightarrow 1$, and $f_{\phi}(\mathcal{T}, \xi)$ becomes independent on $\mathcal{T}$ :

$$
\begin{aligned}
f_{\phi}(\mathcal{T}, \xi) & =\int_{0}^{2} d z S_{\phi}(z) \int_{0}^{\infty} d x \int_{0}^{2 \pi} \frac{d \psi}{2 \pi} \frac{(z \cos \psi-x)^{2}}{x y^{4}} \frac{1}{\Theta_{\xi}^{2}(x) \Theta_{\xi}^{2}(y)} \\
& =c_{I V}^{\eta} \xi^{-2 \eta}
\end{aligned}
$$


where $c_{I V}^{\eta}=\eta U^{\eta} W^{\eta} \int_{0}^{2} d z S_{\phi}(z) z^{2 \eta-2}$. Here, with $J_{n}(u)$ being the Bessel function of the first kind, $U^{\eta} \equiv$ $\int_{0}^{\infty} u^{-2 \eta} J_{1}(u) d u=\frac{4^{-\eta} \Gamma(1-\eta)}{\Gamma(1+\eta)}$, and $W^{\eta} \equiv\left(\int_{0}^{\infty} u^{\eta-1} J_{0}(u) d u\right)^{2}+\left(\int_{0}^{\infty} u^{\eta-1} J_{2}(u) d u\right)^{2}=\left(\frac{2^{\eta-1} \Gamma(\eta / 2)}{\Gamma(1-\eta / 2)}\right)^{2}+$ $\left(\frac{2^{\eta-1} \Gamma(1+\eta / 2)}{\Gamma(2-\eta / 2)}\right)^{2}$. For $\eta=0.8$, this yields $U^{\eta}=1.63$ and $W^{\eta}=2.42$. Eq. (32) describes the asymptotic behavior of the function $f_{\phi}(\mathcal{T}, \xi)$ in region IV.

As a result, we see that the function $f_{\phi}(\mathcal{T}, \xi)$ for large $\xi$ evolves with growing $\mathcal{T}$ as follows (regions of applicability can be easily read off from these equations):

$$
c_{I I} \mathcal{T} \rightarrow c_{I}^{\eta} \xi^{-\frac{3 \eta}{2-\eta / 2}} \mathcal{T}^{\frac{2-2 \eta}{2-\eta / 2}} \rightarrow c_{I V}^{\eta} \xi^{-2 \eta}
$$

As indicated in the main text, the matching of different asymptotes gives us a separation between different regions of the dephasing rate on the $(T-\mu)$ plane: The black line in the Fig. $3 \mathrm{~m}$ corresponds to matching of the first pair of asymptotes. The location of the region IV is related to matching of the second pair.

Small $\xi$

For small $\xi \ll 1$, there are two asymptotic regions depending on the value of $\mathcal{T}$. For $\mathcal{T} \ll 1$, the integral in Eq. (29) is always dominated by the quadratic spectrum $(b=0)$, and function $f_{\phi}(\mathcal{T}, \xi)$ acquires the asymptote linear in $\mathcal{T}$ (compare with Eq. $(30)$ at $b=0)$ :

$$
f_{\phi}(\mathcal{T}, \xi)=c_{I I} \mathcal{T}
$$

At large $\mathcal{T} \gg 1$, the golden rule is applicable. In this region,

$$
f_{\phi}(\mathcal{T}, \xi)=c_{I I I} \log 1 / \xi
$$

where $c_{I I I}=\int_{0}^{2} S_{\phi}(z) / z^{2} d z$. Note, that $c_{I}^{\eta}, c_{I I}, c_{I I I}$ and $c_{I V}^{\eta}$ implicitly depend on $g_{e}$ via $S_{\phi}(z)$, see Eq. (19). Thus, in the case of small $\xi, f_{\phi}(\mathcal{T}, \xi)$ evolves with growing $\mathcal{T}$ as follows

$$
c_{I I} \mathcal{T} \rightarrow c_{I I I} \log 1 / \xi
$$

The blue line in the Fig. 3m corresponds to matching of the above asymptotes.

Equation (24) allows for straightforward numerical evaluation. Here as an illustration, we present the numerical calculation of the function $f_{\phi}(\mathcal{T})$. From the plot we see an excellent agreement with theoretical calculation for the region $\mathcal{T} \ll 1$ and $\mathcal{T} \gg 1$, even for an intermediate value of $\xi=0.52$.

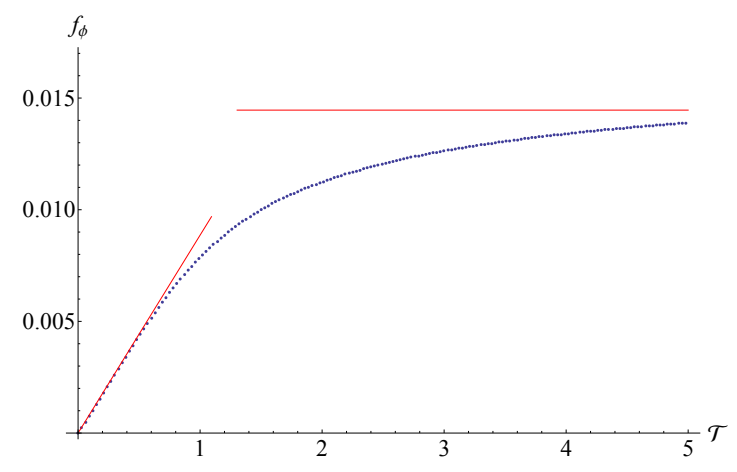

FIG. 3: Plot of the function $f_{\phi}(\mathcal{T})$. The parameters are taken as follows: $\eta=0.8, \xi=0.52$, and $Z=3$. The blue dots are numerical calculation and the red lines are the asymptotes for different region mentioned above.

\section{Phase diagram of the dephasing rate}

In Fig. 3m, the blue and black lines have been found by matching the asymptotic behavior of the dephasing rates deep in regions I, II and III. The blue line is found as a consequence of matching Eq. (34) with Eq. (35). It is defined 
by the criterion $\omega_{k_{F}} \tau_{\phi} \approx 3$, where $\tau_{\phi}$ is calculated numerically from Eq. (11m) with the phonon frequency $\omega_{k_{F}}$ taken from Eq. $(4 \mathrm{~m})$. The black line is found by matching the first pair in Eq. (33), and it can be obtained according to the equation $\omega_{q_{c}} \tau_{\phi} \approx 7$. As mentioned in the main text, the obtained rate has a transition from $\tau_{\phi}^{-1} \sim T^{2}$ to $\tau_{\phi}^{-1} \sim T$ when temperature is increased. This transition is illustrated on Fig. 4.

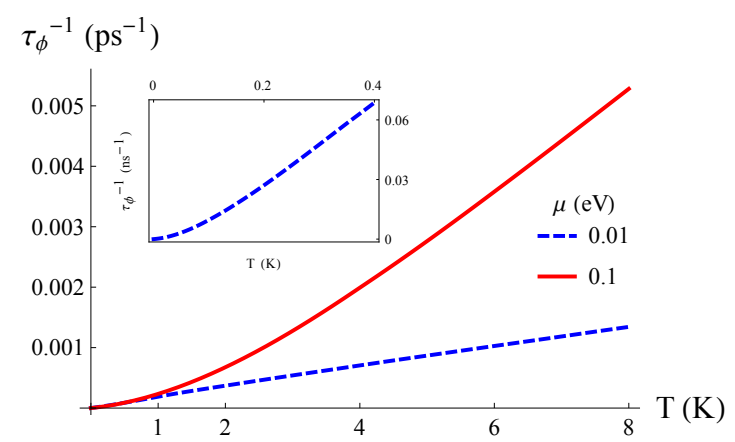

FIG. 4: Depahsing rate as a function of temperature at two different chemical potentials. The transition from a quadratic to linear dependence takes place at $\sim 4 \mathrm{~K}$ for the red solid line. For lower chemical potential (represented by the blue dashed line), the transition happens at a lower temperature as shown in the inset.

Note that besides the regions discussed in the main text, on the $(T-\mu)$ plane there exists another asymptotic region (IV), not mentioned in Eqs. (12m). It lies at very small densities below the blue line and above the line $\xi=1$. In this region, the dephasing rate is still described by the GR, but contrary to region III, renormalization of the FP spectrum at low momenta is essential. In this situation, the dephasing rate equals $\tau_{\phi}^{-1} \sim \gamma T\left(\mu / \mu_{0}\right)^{2 \eta-1}\left(T / T_{0}\right)^{1-\eta}$. However, the relevant densities are so small, that this region does not fit into the scale of Fig. $3 \mathrm{~m}$.

It is instructive to compare the situation with flexural phonons to that for ordinary phonons in semiconductors, where the dephasing process can be viewed as coming from the energy diffusion via low-energy (i.e., quasi-elastic) collisions [4]. Under these conditions, the dephasing rate $\tau_{\phi}^{-1} \sim \sqrt{\left(\tau_{\phi} / \tau_{\text {out }}\right) \delta \epsilon^{2}}$, where $\delta \epsilon$ denotes the characteristic energy transfer during a single scattering event. Thus, the accumulation of phase in the course of the energy diffusion yields $\tau_{\phi}^{-1} \sim\left(\delta \epsilon^{2} / \tau_{\text {out }}\right)^{1 / 3}$. The specific point of our problem, compared to dephasing via usual phonons, is that an electron is coupled to two flexural phonons, and dephasing cannot be described by the energy diffusion process. Because of this two-phonon interaction, the support of the correlation function of the fluctuations is not characterized by any typical frequency $\Omega$, and frequency transfer occurs in such a way that typical energy transfer is of the order of $\tau_{\phi}^{-1}(T)$. In particular, this allows for an energy transfer exceeding $T_{B G}$, although the momentum transfer is limited by $2 k_{F}$. As a result, the decay function $F_{\phi}(t)$ at short times (i.e., in the non-GR regime) is proportional to $t^{2}$, rather than $t^{3}$ as for the case of the energy diffusion. Note that the energy diffusion corresponds to the expansion of the factor $\mathcal{C}^{\phi}(t) \sim(\Omega t)^{2}$ which leads to the $t^{3}$-dependence of $F_{\phi}(t)$. As we have already mentioned, in the case of FPs one cannot expand $\mathcal{C}^{\phi}(t)$.

\section{Vector potential coupling}

Intervalley channel

The similarity (compare Eq. (24) with Eq. (26)) with the scalar potential case makes the calculation of asymptotes for the function $f_{A}^{l}(\mathcal{T}, \xi)$ straightforward. The function $f_{A}^{l}(\mathcal{T}, \xi)$ evolves with the growth of $\mathcal{T}$ in the following fashion: for $\xi \gg 1$,

$$
d_{I I} \mathcal{T} \rightarrow d_{I}^{\eta} \xi^{-\frac{3 \eta}{2-\eta / 2}} \mathcal{T}^{\frac{2-2 \eta}{2-\eta / 2}} \rightarrow d_{I V}^{\eta} \xi^{-2 \eta}
$$

while for $\xi \ll 1$,

$$
d_{I I} \mathcal{T} \rightarrow d_{I I I} \xi^{-1} .
$$

Note that due to the absence of screening, dependence on $\xi$ is stronger than in the case of the scalar potential, compare Eq. (36) with Eq. (38). 
Let us present some technical details. Here $d_{I}^{\eta} \equiv d(-\eta / 2)$, where $d(b)=\frac{\pi}{4} \frac{M_{b}}{1+b / 2}$ and $M_{b}$ is defined in Eq. (31); specifically, for $b=0, d_{I I} \equiv d(0)=1 / 64$. In the GR region, the function $f_{A}^{l}(\mathcal{T}, \xi)$ is independent on $\mathcal{T}$. Its asymptotic behavior is given as follows:

$$
\begin{aligned}
f_{A}(\xi) & =\int_{0}^{2} d z S_{A}(z) \int_{0}^{\infty} d x \int_{0}^{2 \pi} \frac{d \psi}{2 \pi} \frac{1}{x y^{2}} \frac{1}{\Theta_{\xi}^{2}(x) \Theta_{\xi}^{2}(y)} \\
& \equiv\left\{\begin{array}{ll}
d_{I I I} \xi^{-1} & \text { for } \xi \ll 1 \\
d_{I V}^{\eta} \xi^{-2 \eta} & \text { for } \xi \gg 1
\end{array} .\right.
\end{aligned}
$$

Here, for $\eta>1 / 2$ (which is important for the convergence of the following integral),

$$
d_{I I I}=\frac{1}{2} \int_{0}^{\infty} d z \int_{0}^{\infty} d x \int_{0}^{2 \pi} \frac{d \psi}{2 \pi} \frac{1}{x y^{2}} \frac{1}{\Theta_{\xi=1}^{2}(x) \Theta_{\xi=1}^{2}(y)}
$$

and

$$
d_{I V}^{\eta}=2 \eta U^{\eta} V^{\eta} G_{A}
$$

where $U^{\eta}$ is the same as in the case of the scalar potential, $V^{\eta} \equiv\left(\int_{0}^{\infty} u^{\eta-1} J_{0}(u) d u\right)^{2}=\left(\frac{2^{\eta-1} \Gamma(\eta / 2)}{\Gamma(1-\eta / 2)}\right)^{2}$ and $G_{A}=$ $\int_{0}^{2} d z S_{A}(z) z^{2 \eta-2}=\frac{4^{\eta} \sqrt{\pi}}{8} \frac{\Gamma(\eta-1 / 2)}{\Gamma(\eta)}$. For $\eta=0.8$, this yields $V^{\eta}=1.68$ and $G_{A}=1.73$.

\section{Intravalley channel}

For the intravalley Cooperon, the function $f_{A}^{l}(\mathcal{T}, \xi)$ involves $\Xi_{+}$which does not vanish in the limit of $\mathcal{T} \rightarrow 0$. Therefore, it evolves with the growth of $\mathcal{T}$ (i.e., from $\mathcal{T} \ll 1$ to $\mathcal{T} \gg 1$ ) as

$$
2 f_{A}(\xi) \rightarrow f_{A}(\xi)
$$

where $f_{A}(\xi)$ is defined in Eq. (39). It is noteworthy that the static deformations produce dephasing here. This happens because the electrons on the interfering trajectories are coupled to the vector potential of the same sign. The situation is similar to that for a particle in the random magnetic field [5, 6]. Unfortunately, the observation of this effect is obscured by the gaps inevitably produced in these channels by disorder scattering. The rate obtained from $F_{A}^{l}(t)=1$ changes from $2 \tau_{*, A}^{-1}$ to $\tau_{*, A}^{-1}$ with increasing temperature, where

$$
\tau_{*, A}^{-1}=\gamma T\left\{\begin{array}{cc}
2.6\left(\mu / \mu_{0}^{\prime}\right)^{2 \eta-1}\left(T / T_{0}^{\prime}\right)^{1-\eta} & \text { for } \xi \gg 1 \\
1.9 \sqrt{T / T_{0}^{\prime}} & \text { for } \xi \ll 1
\end{array} .\right.
$$

Here $\gamma \sim 0.02$ is the adiabatic parameter defined in the main text, $\mu_{0}^{\prime} \sim \gamma \Delta_{c}$ and $T_{0}^{\prime} \sim \gamma^{2} \Delta_{c}$ where $\Delta_{c}$ describes the energy scale of the anharmonicity (see the main text). Note that for $\xi \ll 1$, the characteristic momentum transfer is $\sim q_{c}\left(\equiv \frac{\sqrt{T \Delta_{c}}}{v_{F}}\right)$ instead of $k_{F}$. As a result, the rate is independent of the chemical potential. Note also that for the intravalley channels the diffusive limit $\left(q_{c} l \ll 1\right)$ for the calculation of dephasing due to the el-FP interaction can be achieved at higher temperature as compared to the intervalley channels. For example, for dimensionless sheet conductance $g_{\square} \sim 10$ and chemical potential $\mu \sim 0.1 \mathrm{eV}$, the diffusive limit occurs already below $T \sim 0.5 \mathrm{~K}$.

[1] J. von Delft, F. Marquardt, R. A. Smith, and V. Ambegaokar, Phys. Rev. B 76, 195332 (2007).

[2] E. McCann, K. Kechedzhi, V. I. Fal'ko, H. Suzuura, T. Ando, and B. L. Altshuler, Phys. Rev. Lett. 97, 146805 (2006).

[3] F. Marquardt, J. von Delft, R. A. Smith, and V. Ambegaokar, Phys. Rev. B 76, 195331 (2007).

[4] B. L. Altshuler, A. G. Aronov, and D. E. Khmelnitsky, Solid State Communications 39, 619 (1981).

[5] A. G. Aronov, A. D. Mirlin, and P. Wölfle, Physical Review B 49, 16609 (1994).

[6] A. G. Aronov and P. Wölfle, Phys. Rev. B 50, 16574 (1994). 